\title{
Selenium Nanoparticles by Moderating Oxidative Stress Promote Differentiation of Mesenchymal Stem Cells to Osteoblasts
}

This article was published in the following Dove Press journal: International Journal of Nanomedicine

\author{
Sabiha Fatima (1) \\ Rawan Alfrayh (iD) \\ May Alrashed ${ }^{2}$ \\ Sarah Alsobaiel \\ Rehan Ahmad (D) ${ }^{3}$ \\ Amer Mahmood (iD) 4 \\ 'Department of Clinical Laboratory \\ Sciences, College of Applied Medical \\ Sciences, King Saud University, Riyadh \\ I I433, Saudi Arabia; ${ }^{2}$ Chair of Medical \\ and Molecular Genetics Research, \\ Department of Clinical Laboratory \\ Sciences, College of Applied Medical \\ Sciences, King Saud University, Riyadh \\ I I433, Saudi Arabia; ${ }^{3}$ Colorectal \\ Research Chair, Department of Surgery, \\ King Saud University, College of \\ Medicine, Riyadh II472, Saudi Arabia; \\ ${ }^{4}$ Stem Cell Unit, Department of \\ Anatomy, College of Medicine, King \\ Khalid University Hospital, King Saud \\ University, Riyadh II46I, Kingdom of \\ Saudi Arabia
}

Purpose: Redox homeostasis plays an important role in the osteogenic differentiation of human mesenchymal stem cells (hMSCs) for bone engineering. Oxidative stress (OS) is believed to induce osteoporosis by changing bone homeostasis. Selenium nanoparticles (SeNPs), an antioxidant with pleiotropic pharmacological activity, prevent bone loss. However, the molecular mechanism underlying the osteogenic activity during hMSCSeNP interaction is unclear.

Methods: This study assessed the effects of different concentrations $(25,50,100$, and 300 $\mathrm{ng} / \mathrm{mL}$ ) of SeNPs on the cell viability and differentiation ability of human embryonic stem cell-derived hMSCs. In addition, we analyzed OS markers and their effect on mitogenactivated protein kinase (MAPK) and Forkhead box O3 (FOXO3) during osteogenesis.

Results: SeNPs increased the cell viability of hMSCs and induced their differentiation toward an osteogenic over an adipogenic lineage by enhancing osteogenic transcription and mineralization, while inhibiting Nile red staining and adipogenic gene expression. By preventing excessive reactive oxygen species accumulation, SeNPs increased antioxidant levels in hMSCs undergoing osteogenesis compared to untreated cells. In addition, SeNPs significantly upregulated the gene and protein expression of phosphorylated c-Jun N-terminal kinase (JNK) and FOXO3a, with no significant change in the expression levels of extracellular signal-related kinase (ERK) and p38 MAPK.

Conclusion: The results approved that low concentrations of SeNPs might enhance the cell viability and osteogenic potential of hMSCs by moderating OS. Increased JNK and FOXO3a expression shows that SeNPs might enhance osteogenesis via activation of the JNK/FOXO3 pathway. In addition, SeNP co-supplementation might prevent bone loss by enhancing osteogenesis and, thus, can be an effective candidate for treating osteoporosis through cellbased therapy.

Keywords: selenium nanoparticles stem cells, antioxidant, osteogenic differentiation

\section{Introduction}

Osteoporosis is one of the most common bone diseases related to aging. It is characterized by structural deterioration of bone tissue. ${ }^{1}$ Oxidative stress (OS) plays an important role in decreasing osteogenic differentiation in cell lines and the bone mineral density during age-related osteoporosis., ${ }^{2,3}$ Plasma antioxidant levels significantly decrease in women with age-related osteoporosis. ${ }^{4}$ Mesenchymal stem cells (MSCs) can induce repair processes at sites of injury in vivo by replenishing bone cells, including chondrocytes and osteoblasts. ${ }^{5,6}$ The

Amer Mahmood

Stem Cell Unit, Department of Anatomy,

College of Medicine, King Saud University,

Riyadh I I46 I, Kingdom of Saudi Arabia

Tel +966- I I-467942 I

Fax +966-II-4693738

Email ammahmood@ksu.edu.sa 
infinite self-renewal capacity and greater differentiation potential of pluripotent embryonic stem cells (ESCs) have enabled their application in biology and medicine. ${ }^{7-9}$ For many clinical applications, in vitro expansion of MSCs is required to obtain high yields of functionally viable cells before transplantation. ${ }^{10}$ Unfortunately, the in vitro expansion of human mesenchymal stem cells (hMSCs) in long-term cell culture leads to a gradual decline in the viability and multipotency of MSCs, which is a major obstacle in cell-based therapy. ${ }^{11,12}$

In long-term cell culture, OS is a potential source of cellular damage, affecting the in vitro expansion and longevity of MSCs, with a decreased number of functionally viable cells for clinical applications. ${ }^{13,14}$ During cell differentiation, increased mitochondrial biogenesis and a metabolic shift toward oxidative phosphorylation change the redox status and spontaneously increase reactive oxygen species (ROS) generation. ${ }^{15}$ Many tissue regenerationbased studies have reported that the most transplanted MSCs undergo OS due to excessive ROS generated by either host tissues or the MSCs themselves. ${ }^{16}$ After several days, this increase in ROS levels leads to cell cycle arrest and the death of transplanted MSCs, and only a small percentage of transplanted MSCs survive, which is insufficient for replacing lost tissue. ${ }^{17}$ High ROS levels can lead to cellular damage and dysfunction, while physiological $/$ moderate ROS levels can improve cell survival, proliferation, and differentiation. ${ }^{18}$ Therefore, ROS regulation by an antioxidant can improve the viability and differentiation of MSCs, which might augment the MSC treatment efficiency in reversing age-related bone loss by osteogenic progenitors. ${ }^{19,20}$

Selenium (Se) is an essential trace element that protects cells against oxidative damage. Se is also required for regulating bone cell proliferation and differentiation. ${ }^{21}$ Nanosized $\mathrm{Se}$ is a highly effective molecular compound with higher antioxidant activity and lower toxicity compared to ordinary Se. ${ }^{22}$ Although the potential use of nanomaterials in regulating MSC differentiation is well understood, the role of selenium nanoparticles (SeNPs) in governing MSC differentiation toward an osteogenic lineage is unclear. Therefore, this study investigated the effect of SeNPs on the cell viability and osteogenesis of human embryonic stem cell (hESC)-derived hMSCs. We also analyzed the effect of SeNPs on intracellular OS markers. In addition, we investigated the expression of the OS-regulated mitogenactivated protein kinases (MAPKs) p38, c-Jun N-terminal kinase (JNK), and extracellular signal-related kinase (ERK), in addition to Forkhead box O3a (FOXO3a), during osteogenesis.

\section{Materials and Methods Cell Culture}

We purchased hESC-derived hMSCs (P3) from Merck Millipore (Burlington, MA, USA) and cultured them in stromal medium containing Dulbecco's Modified Eagle Medium (DMEM; Thermo Fisher Scientific, Waltham, MA, USA) supplemented with $10 \%$ fetal bovine serum (FBS; Thermo Fisher Scientific), 1x penicillinstreptomycin (Thermo Fisher Scientific), $110 \mathrm{mg} / \mathrm{L}$ of sodium pyruvate (Thermo Fisher Scientific), $4 \mathrm{mM}$ L-glutamine (Thermo Fisher Scientific), and $4500 \mathrm{mg} / \mathrm{L}$ of D-glucose (Thermo Fisher Scientific) in a humidified tissue culture incubator at $37^{\circ} \mathrm{C}$ in a $5 \% \mathrm{CO}_{2}$ atmosphere. The cultured hESCs were used for experiments after $80 \%$ confluence.

\section{Characterization of SeNPs}

SeNPs $(5-50 \mathrm{~nm})$ were purchased from Nanocs Inc. (Boston, MA, USA), separated by centrifugation, and redispersed in aqueous medium by sonication. Their shape and size were examined using transmission electron microscopy (TEM) at $200 \mathrm{kV}$ (JEM-2100F; JEOL, Tokyo, Japan). In addition, their fingerprint or composition was measured by Fourier transform infrared (FTIR) spectroscopy in the wavenumber range of $4000-400 \mathrm{~cm}^{-1}$.

\section{Uptake of SeNPs by MSCs Visualized Through Fluorescent Microscopy}

To observe the cellular uptake of SeNPs, we incubated hMSCs with fluorescently labeled SeNPs for $4 \mathrm{~h}$ and then prefixed them with $2.5 \%$ glutaraldehyde in phosphatebuffered saline (PBS) at $4^{\circ} \mathrm{C}$ overnight. Subsequently, we washed the cells thrice with PBS, postfixed them for 45 min with $1 \%$ osmium dissolved in PBS, and washed them by dipping them in water for $10 \mathrm{~min}$. Once the cells were dehydrated, we observed them under a fluorescent microscope to see in the internalization of SeNPs by MSCs.

\section{Cell Viability Assay}

We seeded hMSCs in 96-well culture plates at a density of $2 \times 10^{4}$ cells/well and incubated them for 9 days. After incubation, we added different concentrations $(25,50,100$, and $300 \mathrm{ng} / \mathrm{mL}$ ) of SeNPs to the wells and further 
incubated the cells for 3, 7, and 9 days. Untreated cells were used as the control group. Next, we measured the cell viability on days $0,3,7$, and 9 using alamarBlue assay (Bio-Rad AbD Serotec, USA). Briefly, we added $10 \mu \mathrm{L}$ of alamarBlue substrate to the cultured hMSCs and incubated the plates in the dark at $37^{\circ} \mathrm{C}$ for $2 \mathrm{~h}$. We measured fluorescence levels at excitation and emission wavelengths of 530 and $590 \mathrm{~nm}$, respectively. The fluorescence intensity was proportional to the number of viable cells.

\section{Differentiation of hMSCs into an Osteogenic Lineage}

We performed alkaline phosphatase (ALP) activity and alizarin red (AR)-staining assays to assess the osteogenic differentiation of hMSCs. To induce osteogenesis, we cultured $\sim 1 \times 10^{4} \mathrm{hMSCs}$ in osteogenic induction supplement (OIS) containing 10\% FB, 1\% penicillin-streptomycin, 50 $\mu \mathrm{g} / \mathrm{mL}$ of L-ascorbic acid (Wako Chemicals, Germany), 10 $\mathrm{mM} \beta$-glycerophosphate, and $10 \mathrm{nM}$ dexamethasone (Sigma-Aldrich, St. Louis, MO, USA). We assayed five experimental groups for ALP staining: (i) hMSCs cultured in OIS only (control group); (ii) hMSCs cultured in OIS + $25 \mathrm{ng} / \mathrm{mL}$ of SeNPs (group 1); (iii) hMSCs cultured in OIS $+50 \mathrm{ng} / \mathrm{mL}$ of SeNPs (group 2); (iv) hMSCs cultured in OIS + $100 \mathrm{ng} / \mathrm{mL}$ of SeNPs (group 3); and (v) hMSCs cultured in OIS $+300 \mathrm{ng} / \mathrm{mL}$ of SeNPs (group 4). In groups $1-4$, we quantified the ALP activity after 7 days of cell differentiation using the ALP activity colorimetric assay kit (BioVision, Inc, CA, USA) according to the manufacturer's instructions. Finally, absorbance was measured at a wavelength of $405 \mathrm{~nm}$.

We detected mineralized matrix nodule formation in hMSCs cultured in OIS with or without different concentrations of SeNPs by performing AR staining; hMSCs cultured in OIS without SeNPs were the control group. Briefly, hMSCs were seeded in a 48 -well tissue culture plate at a density of $5 \times 10^{6}$ cells/well, containing OIS and OIS + SeNPs $(25,50,100$, and $300 \mathrm{ng} / \mathrm{mL})$ for 14 days. After 14 days, the cells were washed with PBS, fixed in $70 \%$ ice-cold ethanol at room temperature for $1 \mathrm{~h}$, and stained with the filtered Alizarin red S (ARS) Staining Kit (ScienCell Research Laboratories, Carlsbad, CA, USA) at room temperature for $20 \mathrm{~min}$. Next, the excess dye was washed away with distilled water, and images of stained mineral deposits were taken using an inverted light microscope. To quantify the ARS staining, we eluted the dye with $10 \%(\mathrm{w} / \mathrm{v})$ cetylpyridinium chloride solution (Sigma-
Aldrich) at room temperature for $20 \mathrm{~min}$ and measured the optical density (OD) at a wavelength of $570 \mathrm{~nm}$. Finally, the mineralization rate was calculated as a percentage using the following equation:

$$
\left[\left(\mathrm{OD}_{\text {sample }}-\mathrm{OD}_{\text {control }}\right) / \mathrm{OD}_{\text {control }}\right] \times 100
$$

\section{Detection and Quantification of}

\section{Adipogenesis}

We assessed the adipogenic differentiation of hMSCs in the absence and presence of SeNPs using Nile red staining, which is used to detect intracellular lipid droplets. We added adipogenetic induction supplements, including 10\% horse serum, 10\% FBS (Sigma-Aldrich), 1\% penicillin-streptomycin, $100 \mathrm{nM}$ dexamethasone, $0.45 \mathrm{mM}$ isobutyl methyl xanthine (Sigma-Aldrich), $3 \mu \mathrm{g} / \mathrm{mL}$ of insulin (Sigma-Aldrich), and SeNPs $(25,50,100$, or $300 \mathrm{ng} / \mathrm{mL}$ ), to the culture medium. After 14 days of adipogenic induction, we washed the hMSCs with PBS, added Nile red directly to the cells ( $5 \mu \mathrm{g} / \mathrm{mL}$ in acetone), and incubated the cells at room temperature for $10 \mathrm{~min}$. After staining, the excess dye was washed away with PBS, and images of stain-detecting intracellular lipid droplets were taken using a fluorescent microscope. To quantify Nile red fluorescent staining, we measured the signal using a Spectra Max/M5 fluorescence spectrophotometer plate reader (Molecular Devices, Sunnyvale, CA, USA) at excitation and emission wavelengths of 485 and $572 \mathrm{~nm}$, respectively. Finally, the adipogenic differentiation was calculated as a percentage using the following equation:

$$
\left[\left(\mathrm{OD}_{\text {sample }}-\mathrm{OD}_{\text {control }}\right) / \mathrm{OD}_{\text {control }}\right] \times 100
$$

\section{Quantitative, Real-Time Reverse Transcription Polymerase Chain Reaction} After 18 days of osteogenic and adipogenic induction of hMSCs incubation with or without $50 \mathrm{ng} / \mathrm{mL}$ of SeNPs, the cells were lysed and total RNA was extracted using the innuPREP RNA Mini Kit (Analytik Jena AG, Jena, Germany). Next, we reverse-transcribed $1 \mu \mathrm{g}$ of RNA into complementary DNA (cDNA) using the HighCapacity cDNA Reverse Transcription Kit (Applied Biosystems, Foster City, CA, USA) and a Labnet Multigene thermal cycler (Labnet International Inc., Edison, NJ, USA) according to the manufacturer's instructions. To measure the expression of different osteogenic pathway-focused genes, including ALP, Runt-related transcription factor 2 (RUNX2), type I collagen (COL1), 
osteocalcin (OCN), osteopontin (OPN), cadherin-11 (CDH11), and osteonectin (SPARC), the samples were then subjected to quantitative, real-time reverse transcription polymerase chain reaction (RT-PCR) analysis using the Power SYBR $^{\circledR}$ Green RNA-to-CT ${ }^{\mathrm{TM}}$ 1-Step Kit (Applied Biosystems). In addition, we detected the gene expression of the master regulator for adipogenesis, peroxisome proliferator-activated receptor-gamma (PPAR- $\gamma$ ), and CCAAT/enhancer-binding protein $\alpha(\mathrm{C} / \mathrm{EBP} \alpha)$. We also analyzed the messenger RNA (mRNA) expression of MAPK and FOXO3 to check its consistency with protein expression. Finally, we expressed the relative expression of the genes of interest normalized to glyceraldehyde 3-phosphate dehydrogenase (GAPDH) as a fold-change. Supplementary Table 1 lists the primer sequences used in this study.

\section{Estimation of Oxidative Stress Markers}

After 3 days of osteogenic differentiation, we measured the intracellular ROS levels using flow cytometry (FACSCalibur; Becton Dickinson, San Jose, CA, USA) on the basis of the fluorescence-associated oxidation of 2',7'-dichlorodihydrofluorescein (DCFH) to $2^{\prime}, 7^{\prime}-$ dichlorofluorescein. Briefly, we added $1.0 \mu \mathrm{M} 2^{\prime}, 7^{\prime}-$ dicholorodihydrofluorescein diacetate (DCF-DA) to harvested hMSCs and incubated them at $37^{\circ} \mathrm{C}$ for $15 \mathrm{~min}$. Next, the cells were washed with PBS, and the ROS levels in the incubated samples were estimated using flow cytometry (BD Biosciences, San Jose, CA, USA). Data were acquired and analyzed using CellQuest pro ver 6.0 (excitation at 488 $\mathrm{nm}$ and detection at $535 \mathrm{~nm}$ ). We also detected reduced glutathione (GSH) levels using the Glutathione Colorimetric Assay Kit (BioVision Inc., CA, USA). Finally, we analyzed superoxide dismutase (SOD) and catalase activities using the Superoxide Dismutase Activity Assay Kit and the Catalase Activity Colorimetric/Fluorometric Assay Kit (Abcam, MA, USA), respectively, according to the manufacturer's instructions.

\section{Detection of MAPK and FOXO3a Expression by Western Blotting}

We performed Western blotting to study the protein expression levels of p-p38, p-ERK1/2, p-JNK, and p-FOXO3a in hMSCs after incubation with or without 50 $\mathrm{ng} / \mathrm{mL}$ of SeNPs during osteogenic differentiation. After 3 days of osteogenic induction, the hMSCs were harvested and washed twice with PBS. Next, cell lysates were prepared in radioimmunoprecipitation assay (RIPA) lysis buffer (Boston Bioproducts, Ashland, MA, USA), the mixture was centrifuged at $14,000 \mathrm{rpm}$ for $15 \mathrm{~min}$, and the supernatant was collected. Subsequently, 10-20 $\mu \mathrm{g}$ of protein estimated using Bradford protein assay was loaded on electrophoresis gels, the separated proteins were transferred to a $0.2 \mu \mathrm{m}$ polyvinylidene difluoride (PVDF) membrane using a turbo protein transfer system (Bio-Rad Laboratories, Hercules, CA, USA), and the blots were blocked using Sea Block blocking buffer (Thermo Fisher Scientific) at room temperature for $1 \mathrm{~h}$. The PVDF membrane was washed with PBS containing $0.1 \%$ Tween-20 (PBST) and incubated overnight at $4{ }^{\circ} \mathrm{C}$ with primary antibodies p-p38, p-ERK1/2, p-JNK, p-FOXO3a (Abcam, MA, USA), and $\beta$-actin (Santa Cruz Biotechnology, CA, USA). Next, the blots were washed and then incubated with horseradish peroxidase (HRP)-conjugated secondary antibodies at $25^{\circ} \mathrm{C}$ for $1 \mathrm{~h}$. Finally, the signals were detected using the Pierce enhanced chemiluminescence (ECL) detection system (Thermo Fisher Scientific), and image analysis was performed using ImageLab software (Bio-Rad Laboratories).

\section{Statistical Analysis}

All data were expressed as means \pm standard deviation (SD). We performed analysis of variance (ANOVA) to test differences between control and experimental groups using GraphPad Prism7 (GraphPad Software Inc., CA, USA). $P<0.05$ was considered statistically significant.

\section{Results}

\section{Characterization and Cellular Uptake of SeNPs}

The SeNPs were characterized to check their stability in terms of their shape, size, and composition. The TEM image (Figure 1A) showed a clear, spherical morphology of the SeNPs, with an average diameter of $20 \pm 5.6 \mathrm{~nm}$ (Figure 1B). The FTIR spectrum, which represented symmetric and asymmetric vibration bands of the SeNPs, showed a broad peak at $3200-3500 \mathrm{~cm}^{-1}$, indicating the presence of the amide $(-\mathrm{NH})$ group, while a strong peak at $1637 \mathrm{~cm}^{-1}$ corresponded to the $\mathrm{C}=\mathrm{O}$ group. ${ }^{23}$ The peak at $767.5 \mathrm{~cm}^{-1}$ was related to the stretching mode of the metal peaks of Se (Figure 1C).

The fluorescence microscopic image of hMSCs cultured in the absence of SeNPs did not show internalized SeNPs (Figure 2A). In contrast, in hMSCs cultured in the presence of $50 \mathrm{ng}$ of SeNPs, the SeNPs were localized 

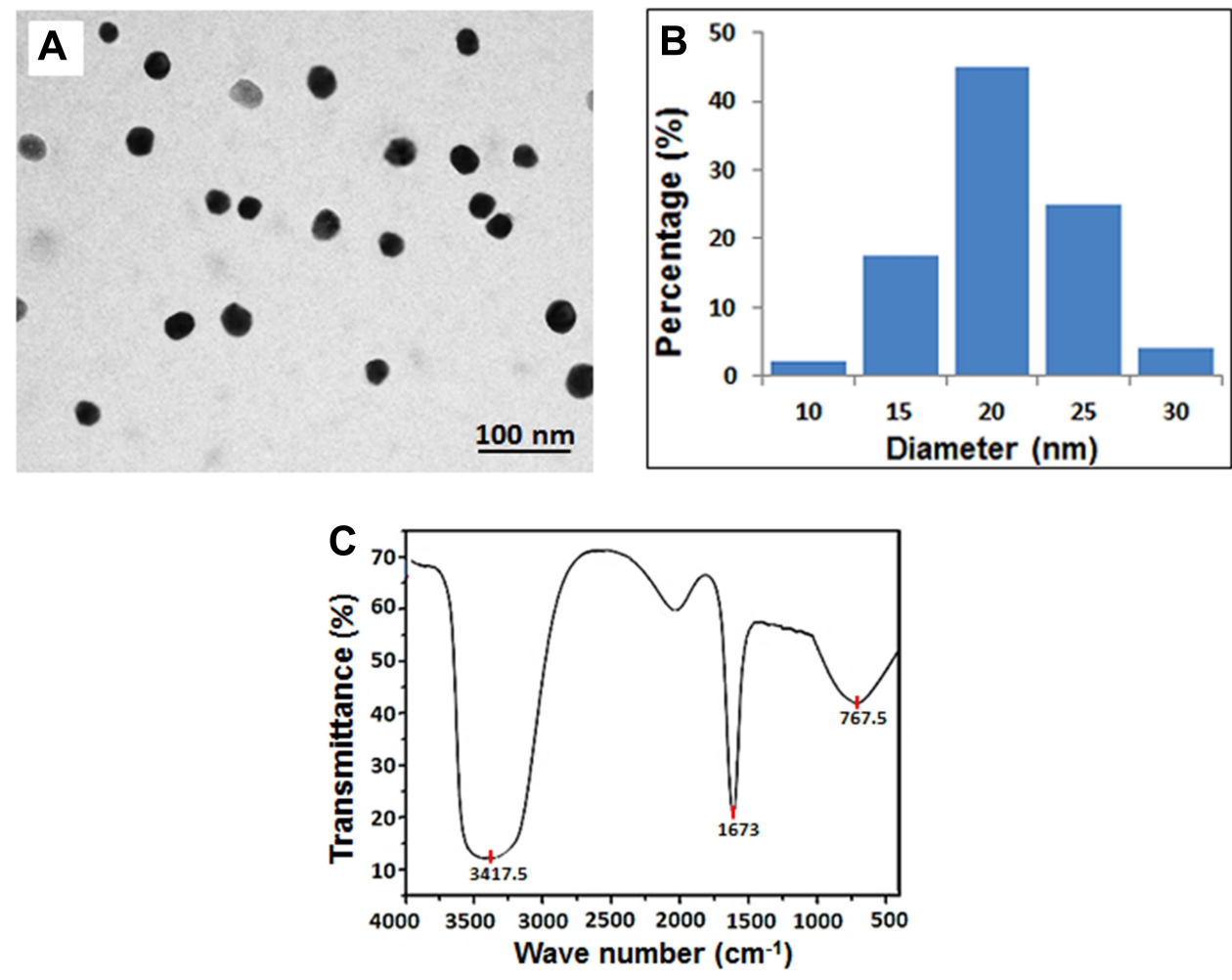

Figure I Characterization of the SeNPs. (A) Transmission electron microscopic image (Scale bar 100 nm); (B) A histogram showing size distribution of the SeNPs; (C) Fourier Transform Infrared spectroscopy (FTIR) of selenium nanoparticles showing the functional characteristics of the nanoparticles.

Abbreviations: FTIR, Fourier transform infrared; SeNPs, selenium nanoparticles.

inside the hMSCs and not merely attached to their surface (Figure 2B and $\mathrm{C}$ ). We also visualized agglomerated SeNPs in a few vesicles in the cytoplasm (Figure 2C).

\section{SeNPs Increase Cell Viability}

Figure 3 shows the fluorescence intensities of untreated (control) and SeNP-treated hMSCs. We observed higher cell viability in hMSCs treated with 25,50 , and $100 \mathrm{ng}$ of SeNPs, while the cell viability significantly decreased at a concentration of $300 \mathrm{ng}$ compared to the control group
$(P<0.001)$. In addition, hMSCs treated with $50 \mathrm{ng}$ of SeNPs showed the highest cell viability $(P<0.001)$ compared to hMSCs treated with 25 and $100 \mathrm{ng}$ of SeNPs.

\section{SeNPs Induce Osteogenic Differentiation}

We determined the effect of SeNPs on osteogenic differentiation of hMSCs cultured for 7 days by measuring their ALP activity in order to evaluate the expression of an early phenotypic marker for osteogenesis (Figure 4A). MSCs treated with SeNPs 25, 50, $100 \mathrm{ng}$ had higher ALP activities

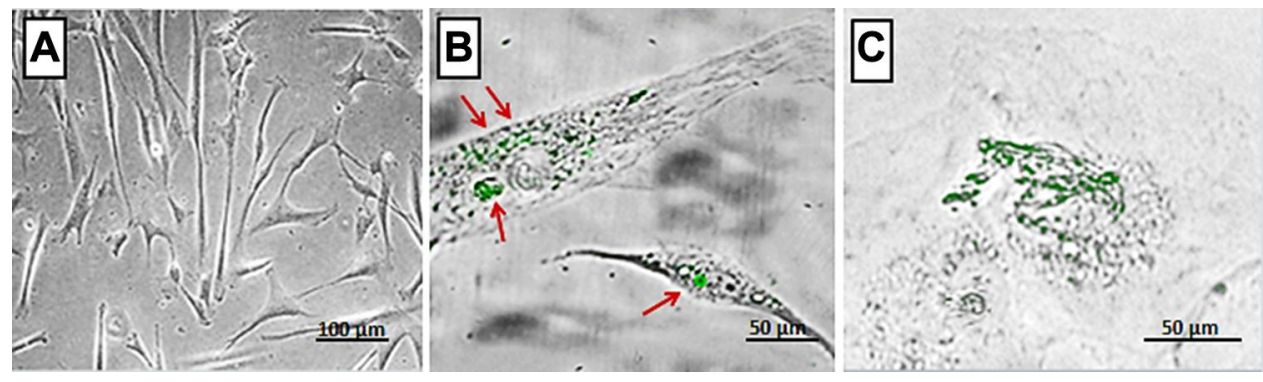

Figure 2 Fluorescence image of cellular internalization of SeNPs $(50 \mathrm{ng} / \mathrm{mL})$ in hMSCs. (A) Control (Scale bar 100 $\mu \mathrm{m})$; (B) Localization of SeNPs ( $\uparrow)$ agglomerates in

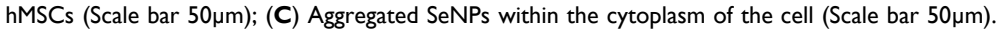

Abbreviations: SeNPs, selenium nanoparticles; hMSCs, human mesenchymal stem cells. 


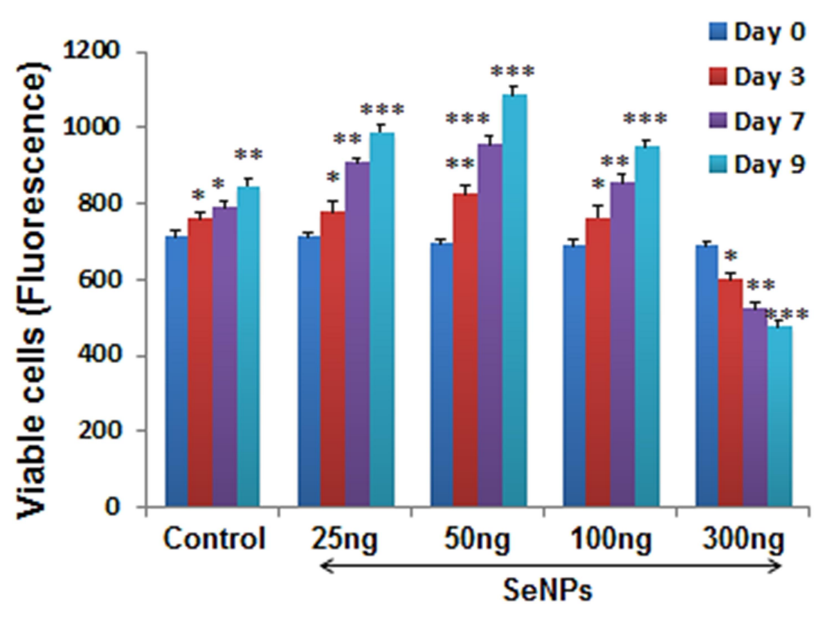

Figure 3 Fluorescence intensity of viable hMSCs treated with different SeNP concentrations using alamarBlue assay at $0,3,7$, and 9 days. Cells treated with lower doses of SeNPs showed significant increase in the number of viable cells from day 0 to day 9 compared to the untreated cells. In contrast, treatment with $300 \mathrm{ng} / \mathrm{mL}$ SeNPs notably decreased the cell viability on day 9 of the cell culture. Results are mean $\pm S D$ of the triplicate experiments, ${ }^{*} p<0.05,{ }^{*} p<0.01, * * * p<0.001$ versus control.

Abbreviations: hMSCs, human mesenchymal stem cells; SeNPs, selenium nanoparticles.

than the control group. The results revealed a considerable $(\mathrm{p}<0.001)$ increase of ALP activity in MSCs treated with SeNPs at a concentration of $50 \mathrm{ng}$. In contrast, ALP activity in MSCs treated with $300 \mathrm{ng}$ of SeNPs significantly decreased compared to the control group.

Mineralized matrix nodule formation, a phenotypic marker of the last stage of mature osteoblasts, was analyzed to assess the progression of hMSCs to an osteogenic lineage. Calcium deposits can specifically be stained a bright orange- red by AR staining. After 14 days of osteogenic induction, hMSCs treated with 25, 50, $100 \mathrm{ng}$ showed increased mineralization nodule formation compared to the control group. In contrast, the cells treated with 300 ng significantly suppressed mineralization nodule formation in hMSCs undergoing osteogenesis (Figure 4B). This indicates that lower concentrations of SeNPs promoted successful hMSC differentiation into osteoblasts. Treatment with SeNPs at a dosage of $50 \mathrm{ng}$ showed maximum $(\mathrm{p}<0.001)$ mineralized nodule formation, compared to the control group.

\section{SeNPs Inhibit Adipogenic Differentiation}

Figure 5A and $\mathrm{B}$ show the concentration-dependent effect of SeNPs on adipogenic differentiation of hMSCs in the presence of adipogenic induction medium, as determined by staining intracytoplasmic lipids with Nile red. SeNPs significantly inhibited adipogenic differentiation of hMSCs compared to the control group. We observed significant inhibition of adipogenic differentiation at a SeNP concentration of $50 \mathrm{ng}$ on day 14 and more decreased adipocyte formation at a SeNP concentration of $300 \mathrm{ng}$ compared to the untreated control group.

\section{SeNPs Lower Oxidative Stress During Osteogenesis}

ROS levels can be used to evaluate the concentrationdependent free-radical-scavenging effect of SeNPs on hMSCs undergoing osteogenic differentiation. In this assay, we measured the intracellular levels of ROS using
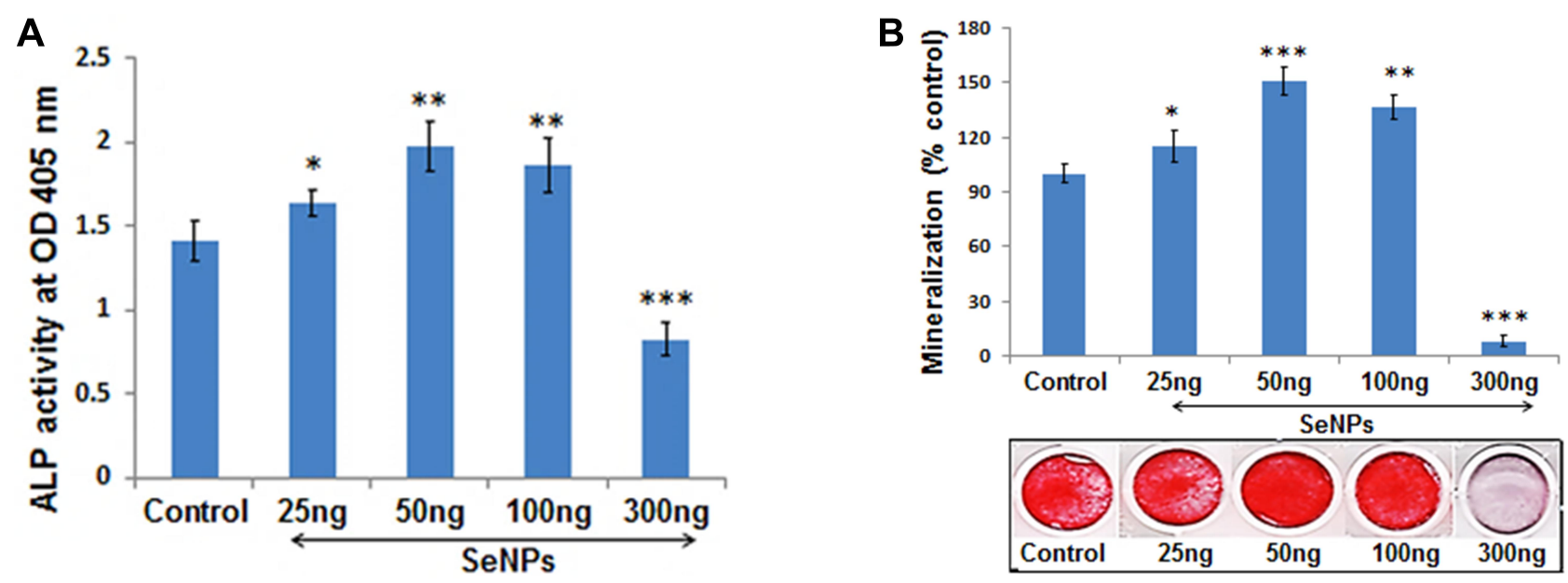

Figure 4 Effect of different SeNP concentrations on osteogenic differentiation of hMSCs. (A) Comparison of the alkaline phosphatase (ALP) activity measured after 7 days of osteogenic induction shows that lower concentrations of SeNPs increased the ALP activity compared to the untreated cells during osteogenesis (B) Quantification of mineralized nodule formation of MSCs visualized by Alizarin red staining after 14 days of osteogenic differentiation shows increased mineralization induced by lower concentration $(25,50,100 \mathrm{ng} / \mathrm{mL})$ of SeNPs. Results are mean \pm SD of the triplicate experiments: ${ }^{*} \mathrm{p}<0.05,{ }^{*} \mathrm{p}<0.01$, $* * * \mathrm{p}<0.001$ versus control.

Abbreviations: SeNPs, selenium nanoparticles; hMSCs, human mesenchymal stem cells. 


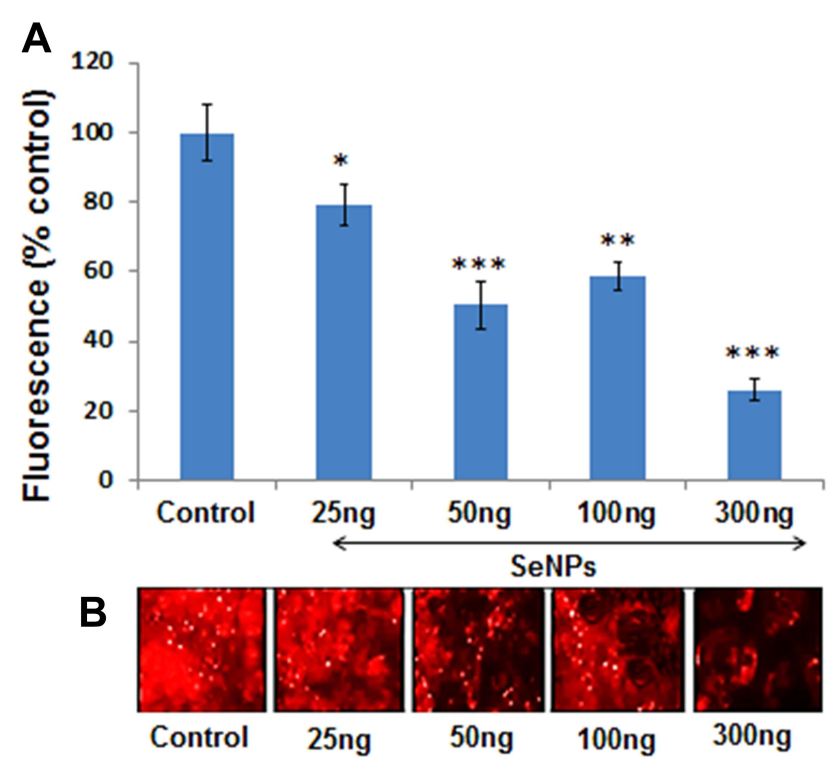

Figure 5 Effect of different SeNP concentrations on adipogenic differentiation of hMSCs. (A) Quantification of Nile red staining represented as percent fluorescence change relative to control at day 14 of adipogenesis shows that SeNPs significantly suppressed the MSCs differentiation to adipogenic lineage. (B) Nile red staining was used to assess the effect of different concentration of SeNPs on adipogenic differentiation of MSCs. Results are mean $\pm S D$ of the triplicate experiments: ${ }^{*} p<0.05$, ** $p<0.01$, **** $p<0.001$ versus control.

Abbreviations: SeNPs, selenium nanoparticles; hMSCs, human mesenchymal stem cells.

DCFH-DA in hMSCs with or without SeNP treatment. DCF fluorescence significantly decreased at SeNP concentration of 25, 50, and $100 \mathrm{ng}$, indicating that ROS generation decreases compared to the control group (Figure 6B). The shift in the histogram (green line) toward the right indicated enhanced ROS scavenging at a SeNP concentration of $50 \mathrm{ng}$ (Figure 6A). In contrast, ROS levels significantly increased at a SeNP concentration of $300 \mathrm{ng}$ compared to control group $(P<0.001)$ (Figure 6A and B).

In addition, lower SeNP concentrations significantly enhanced the level of enzymatic antioxidants, such as SOD and catalase. MSCs treated with $50 \mathrm{ng}$ of SeNPs showed the maximum increase in antioxidant activity compared to the control group $(P<0.001)$, while hMSCs treated with a SeNP concentration of $300 \mathrm{ng}$ significantly inhibited the activity of the enzymatic antioxidants $(P<0.001)$ (Figure 6C).

\section{SeNPs Enhance Osteogenic- and Inhibit Adipogenic-Specific Gene Expression}

On the basis of the concentrated-dependent protective effect of SeNPs, we selected a SeNP concentration of $50 \mathrm{ng} / \mathrm{mL}$ for subsequent experiments. Specific transcription factors regulate the interconversion of hMSCs to a particular lineage with repression of alternative phenotypes. ALP, RUNX2, and COL1a1 determine the osteoblast lineage from pluripotent MSCs, while PPAR- $\gamma$ and $\mathrm{C} / \mathrm{EBP} \alpha$ induce hMSCs to undergo adipocyte differentiation. RT-PCR results showed significantly increased ALP, RUNX2, and COL1al expression and significantly attenuated PPAR- $\gamma$ and $\mathrm{C} / \mathrm{EBP} \alpha$ expression compared to the control group (Figure 7), which is consistent with our observations in cellular assays, indicating that SeNPs preferentially favor hMSC differentiation into an osteogenic over an adipogenic lineage. We also performed RT-PCR to test the transcriptional profile of proteins, such as OCN, OPN, CDH11, and SPARC that significantly contribute to osteoblast differentiation and bone formation. SeNPs markedly upregulated all osteogenesis-promoting genes examined. The transcriptional profile of osteogenic marker genes clearly indicated the effect of SeNPs on osteogenic differentiation, as observed by increased ALP activity (Figure 4A) and mineralized matrix nodule formation (Figure 4B).

\section{SeNPs Modulate JNK and FOXO3a Expression}

We performed Western blotting to evaluate the effect of SeNP treatment on the key proteins of the MAPK pathway and FOXO3a expression during osteogenesis. We analyzed the expression MAPK signaling proteins, including p38, JNK, and ERK (Figure 8A). SeNP treatment significantly increased $\mathrm{p}$-JNK and p-FOXO3a expression, while $\mathrm{p}$-ERK and p-p38 expression did not substantially change compared to the control group (Figure 8B).

In addition, we performed RT-PCR to study the effect of SeNPs on MAPK and FOXO3a gene expression. The transcriptional profile was consistent with protein expression levels (Figure 8C).

\section{Discussion}

In this study, we investigated the effect of supplementation of SeNPs as a potential means to promote osteogenesis of $\mathrm{hESC}$ derived hMSCs in vitro. The hMSCs have a controlled redox status to manage OS. However, in vitro expansion and differentiation disturb the redox status of proliferating hMSCs. ${ }^{24-26}$ Antioxidants, such as SOD and glutathione peroxidase, which are expressed in high levels in hESCs, are downregulated during osteogenic differentiation. ${ }^{24}$ Therefore, in addition to the metabolic shift toward oxidative phosphorylation, antioxidant downregulation might also be associated with increased intracellular ROS levels during osteogenic differentiation. 

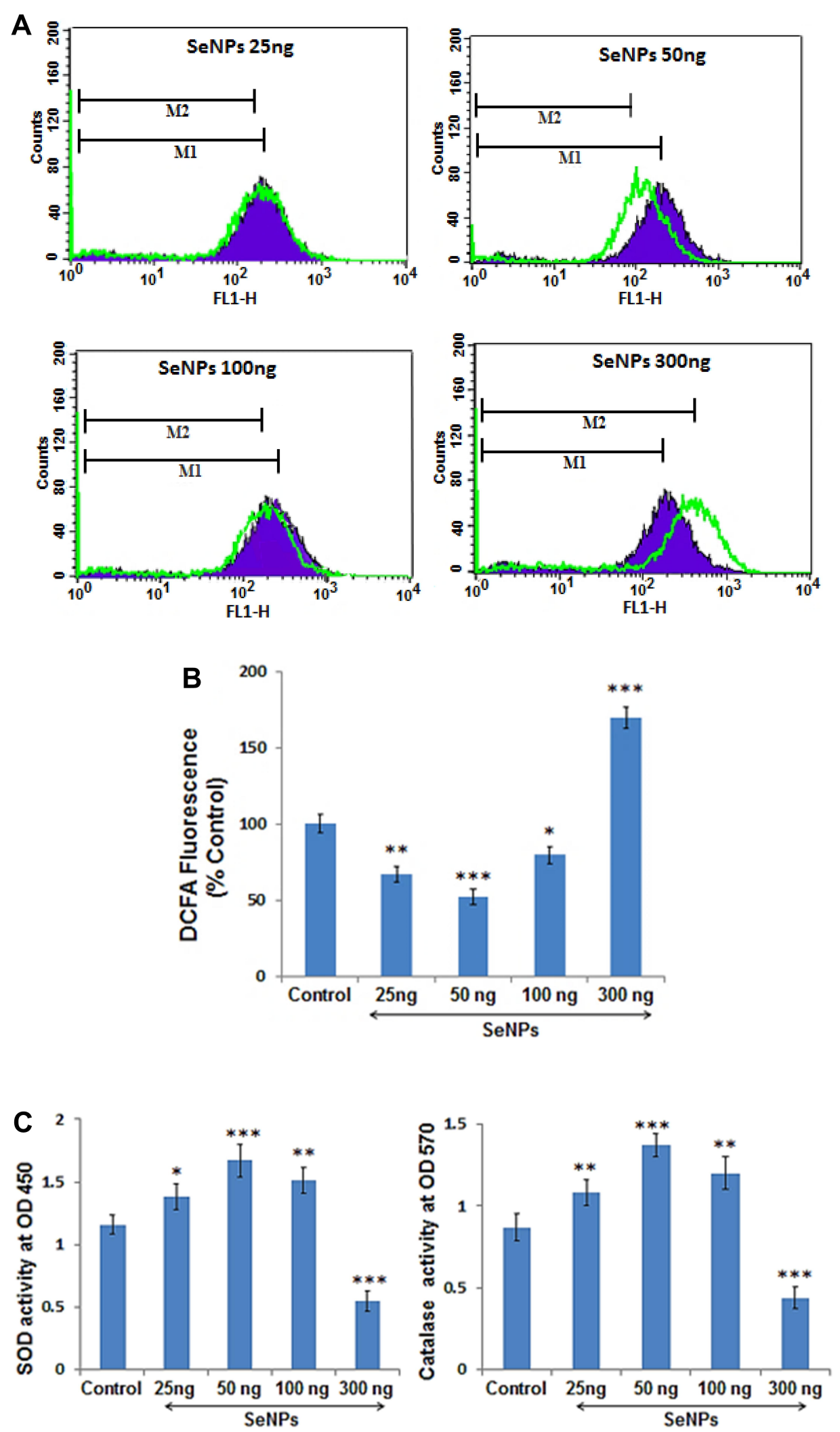

Figure 6 Effects of different SeNP concentrations on OS parameters measured after 3 days of osteogenic induction. (A) Histogram presentation of flow cytometric analysis of ROS levels in control cells (shaded purple) compared to cells treated with different concentration of SeNPs at 50, 100, 300ng/mL respectively (green lines). (B) Quantitative analysis of DCFA fluorescence intensity with respect to the untreated control shows that lower doses of SeNPs significantly reduced the ROS level whereas, high dose resulted in excessive ROS generation during osteogenesis. (C) Absorbance of the enzymatic antioxidants, SOD and Catalase in control and SeNP treated groups represents that lower doses of SeNPs enhanced the oxidative potential of the cells undergoing osteogenesis. Results are mean \pm SD of the triplicate experiments: ${ }^{*} p<0.05$, $* * p<0.01$, ${ }^{* * *} p<0.00$ I versus control. Abbreviations: SeNPs, selenium nanoparticles; OS, oxidative stress; SOD, superoxide dismutase; ROS, reactive oxygen species. 


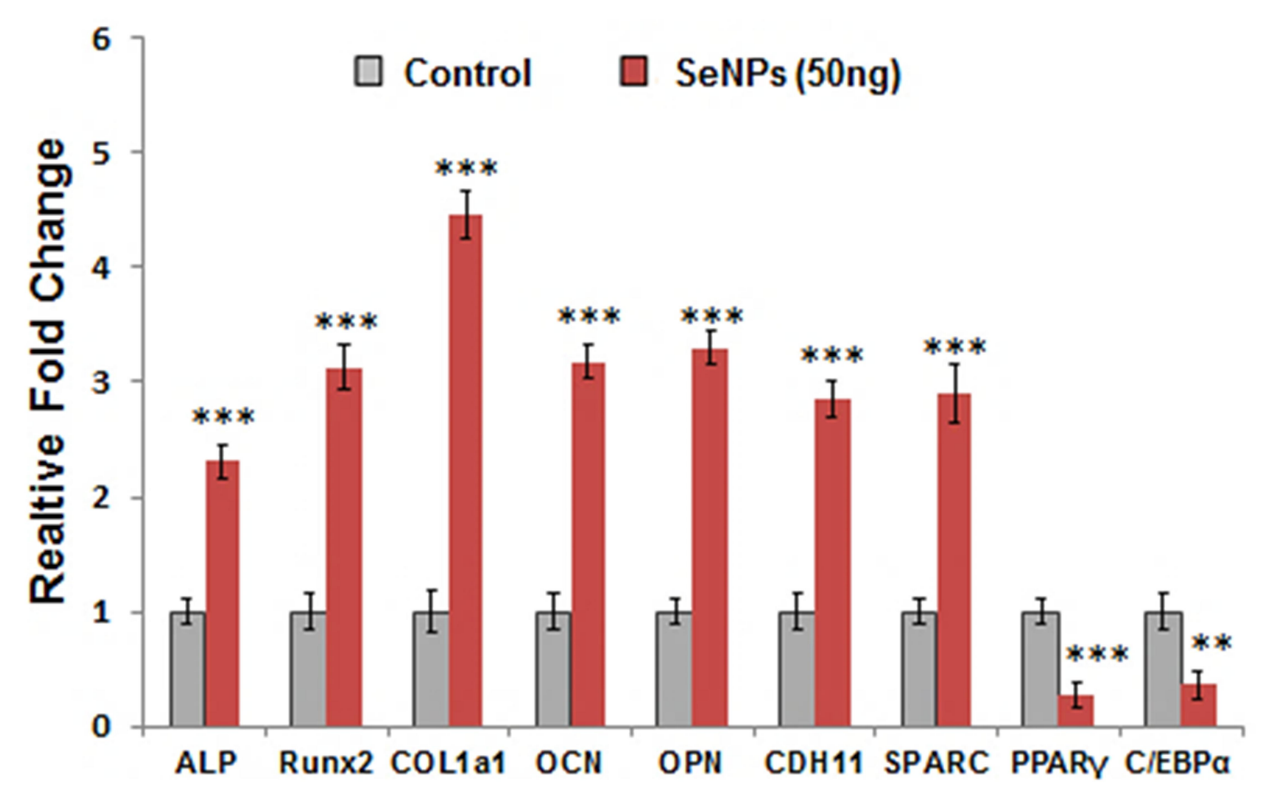

Figure 7 Relative mRNA expression of osteogenic- and adipogenic-specific gene expression in hMSCs upon SeNP treatment (50 ng/mL). SeNPs upregulated the osteogenic and downregulated the adipogenic transcriptional profile of MSCs undergoing cellular differentiation. Results are mean \pm SD of the triplicate experiments: $* * \mathrm{p}<0.01$, *** $\mathrm{p}<0.001$ versus control.

Abbreviations: mRNA, messenger RNA; hMSCs, human mesenchymal stem cells; SeNPs, selenium nanoparticles.

Culturing hMSCs with a low SeNP concentration significantly inhibits ROS levels and increases the antioxidant activity within the cells. Decreased OS is associated with improved cell viability and enhanced differentiation of hMSCs to an osteogenic over an adipogenic lineage. There is an inverse relationship between osteogenic and adipogenic differentiation. ${ }^{27}$ This inverse relationship is indicated by the concurrent upregulation of RUNX-2 (a master regulator of osteogenesis) and downregulation of the adipogenic marker PPAR- $\gamma$ (which induces cells to differentiate into adipocytes). ${ }^{28-30}$ In addition, supplementation with a high $(300 \mathrm{ng} / \mathrm{mL})$ SeNP concentration results in excessive ROS generation with compromised antioxidant defense and decreased cell viability. These findings indicate that SeNPs at supraphysiological levels might change from antioxidants to pro-oxidants, which might induce cytotoxicity. ${ }^{31}$ Therefore, increased cell viability and osteogenesis at low SeNP concentrations could be due to regulation of ROS to controlled or moderate levels in cell culture and the commitment to an osteogenic lineage, with inhibited alternative phenotypes. In addition, increased OS favors hMSC differentiation to adipocytes, indicating that the OS level determines the fate of the cell. $^{32}$
The positive effect of low SeNPs concentrations on osteogenesis is indicated by a significant increase in the activity and gene expression of ALP (an early osteogenic marker) and mineralized matrix nodule formation (a phenotypic marker of mature osteoblasts). ${ }^{33}$ Enhanced gene expression of bone matrix proteins, such as COL1a1, OPN, OCN, and CDH11, which mediate cell-cell adhesion, further indicates osteoblast lineage commitment and differentiation. ${ }^{34}$ Overall, the transcriptional profile of osteogenic marker genes clearly illustrates the positive effect of SeNPs in enhancing osteogenesis, also supported by observations of enhanced ALP activity and mineralized matrix nodule formation (Figure 4A and B).

Incubating cells with nanomaterials in the culture medium enables serum proteins to adsorb to the nanomaterial surface, which induces nanomaterial entry into cells by receptormediated endocytosis. ${ }^{35}$ Light microscopy image shows the presence of fluorescence-labeled SeNPs in the cytoplasm. Once inside a cell, the SeNPs might interact with cytoplasmic proteins, which can regulate the fate of hMSCs through mechanical stimuli-activated signal transduction. ${ }^{9}$ On the basis of these findings, we hypothesized that the MAPK pathway, which is activated by mechanical stresses, might be affected during hMSC-SeNP interaction. To test this 

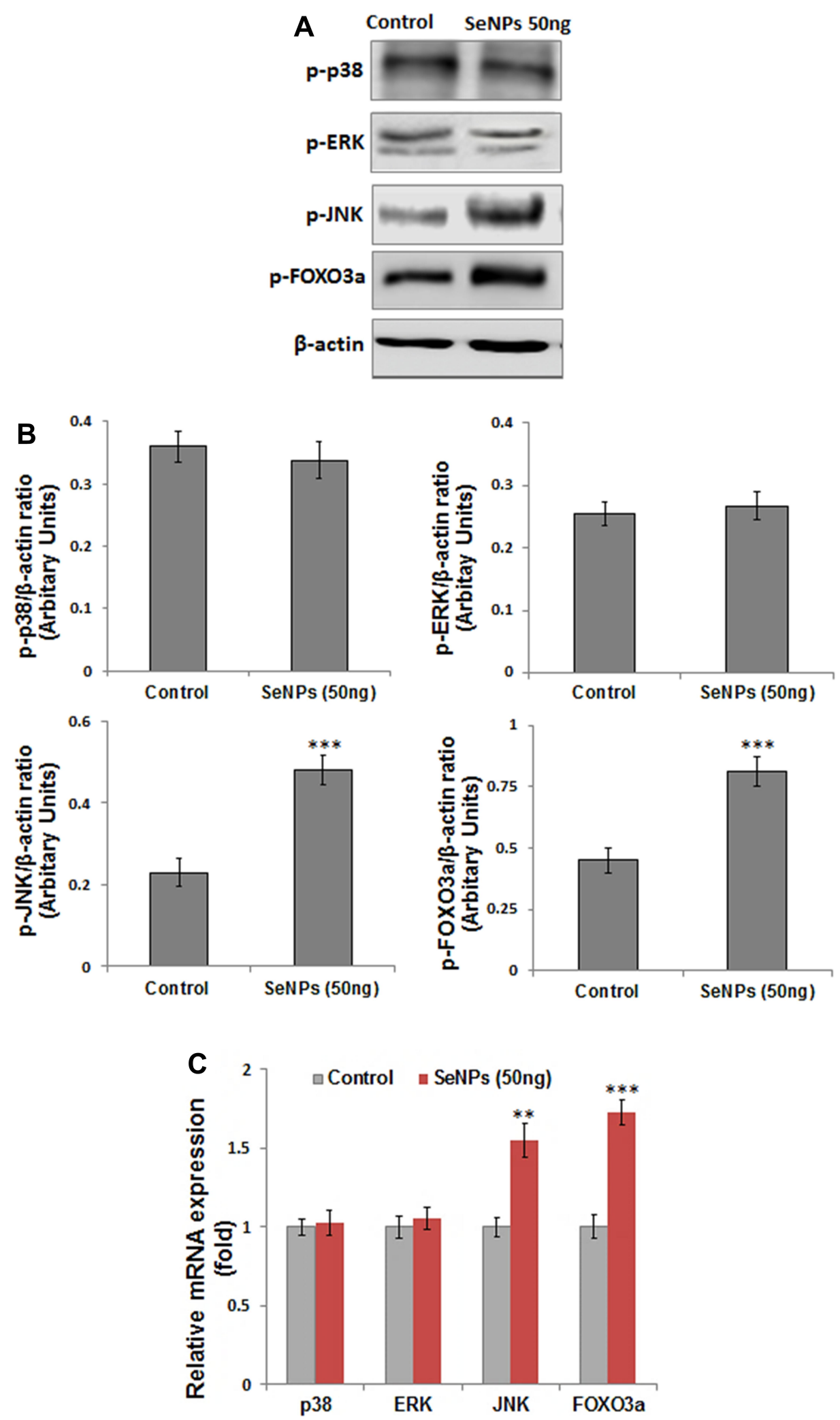

Figure 8 Effects of SeNP (50 ng/mL) on protein and gene expression of p38, ERK, JNK, and FOXO3a measured after 3 days of osteogenic induction. (A) SeNPs significantly enhanced the protein expression of $\mathrm{p}-\mathrm{JNK}$ and $\mathrm{p}$-FOXO3a expression with no notable change in $\mathrm{p}$ - $\mathrm{p} 38$ and $\mathrm{p}$-ERK expression relative to control; $\beta$-actin was used as internal control (B) Density of the protein from Western blot were quantified and expressed as fold change relative to $\beta$-actin. (C) Relative fold change in the mRNA expression of MAPK and FOXO3a gene analyzed by RT-PCR. Results are mean \pm SD of the triplicate experiments: $* * p<0.01$, *** $p<0.00$ I versus control.

Abbreviations: ERK, extracellular signal-related kinase; JNK, c-Jun N-terminal kinase; FOXO3a, Forkhead box O3a; mRNA, messenger RNA; SeNPs, selenium nanoparticles. 
hypothesis, we assessed the involvement of the key proteins of the MAPK pathway by Western blotting and RT-PCR during $\mathrm{hMSC}-\mathrm{SeNP}$ interaction. Increased expression of $\mathrm{p}-\mathrm{JNK}$, a stress-activated kinase, and no substantial change in p-ERK and p-p38 expression indicates that activation of the JNK signaling pathway might be involved in the preferential differentiation of hMSCs to osteoblasts. The JNK-MAPK pathway plays an important role in the OS-coordinated cellular response to different stresses, including ROS, which also play a positive role in modulating osteogenic differentiation. ${ }^{36}$ A mild oxidative environment can activate the pro-survival cellular mechanism of JNK-MAPKs to repair the damage before committing cells to death. ${ }^{37}$ The pro-survival function of JNK depends on the regulation of the antioxidant response. In response to low OS, the activated JNK survival signaling pathway mediates FOXO phosphorylation and translocation from the cytoplasm into the nucleus. ${ }^{38}$ As a transcription factor, FOXO not only enhances the synthesis of antioxidant enzymes Mn-SOD and catalase but also binds to the promoters of RUNX2, ALP, and OCN and assists in commitment to an osteogenic lineage. ${ }^{39,40}$ Therefore, increased gene and protein expression of p-JNK and FOXO3a after SeNP treatment might lead to decreased ROS levels via induction of antioxidant enzymes. Consequently, activation of the JNK/FOXO3a pathway by enhancing cellular antioxidants might improve cell viability and enhance the osteogenic potential of hMSCs, as observed by the increased expression of bone marker proteins in SeNP-treated hMSCs compared to untreated hMSCs.

\section{Conclusion}

A low SeNP concentration $(50 \mathrm{ng} / \mathrm{mL})$ by decreasing OS plays an important role in enhancing the differentiation of hMSCs to an osteogenic lineage. Increased gene and protein expression of JNK and FOXO3a indicates the role of the JNK/FOXO3a pathway in SeNP-induced osteogenesis. This, in turn, indicates that SeNPs due to its antioxidative and osteoinductive properties can be used as potential therapeutic agents in treating osteoporosis through cell-based therapy.

\section{Abbreviations}

MSCs, mesenchymal stem cells; mRNA, messenger RNA; SeNPs, selenium nanoparticles; ESCs, embryonic stem cells; MAPK, mitogen-activated protein kinase; FOXO, Forkhead box O3; ROS, reactive oxygen species; ERK, extracellular signal-related kinase; JNK, c-Jun N-terminal kinase; OS, oxidative stress; hMSCs, human mesenchymal stem cells; DMEM, Dulbecco's Modified Eagle Medium; FBS, fetal bovine serum; TEM, transmission electron microscopy;
FTIR, Fourier transform infrared; PBS, phosphate-buffered saline; OIS, osteogenic induction supplement; ALP, alkaline phosphatase; AR, alizarin red; ARS, Alizarin red S; OD, optical density; cDNA, complementary DNA; RT-PCR, reverse transcription polymerase chain reaction; RUNX2, Runt-related transcription factor 2; COL1, type I collagen; OCN, osteocalcin; OPN, osteopontin; CDH11, cadherin-11; SPARC, osteonectin; $\mathrm{C} / \mathrm{EBP} \alpha, \mathrm{CCAAT} /$ enhancer-binding protein $\alpha$; PPAR-G, peroxisome proliferator-activated receptor-gamma; GAPDH, glyceraldehyde 3-phosphate dehydrogenase; DCFH, 2',7'-dichlorodihydrofluorescein; DCF-DA, 2',7'-dicholorodihydrofluorescein diacetate; GSH, glutathione; SOD, superoxide dismutase; SD, standard deviation; ANOVA, analysis of variance; RIPA, radioimmunoprecipitation assay; PVDF, polyvinylidene difluoride; HRP, horseradish peroxidase; ECL, enhanced chemiluminescence.

\section{Acknowledgment}

This research project was supported by a grant from the "Research Center of the Female Scientific and Medical Colleges", Deanship of Scientific Research, King Saud University.

\section{Author Contributions}

All authors contributed to data analysis, drafting or revising the article, have agreed on the journal to which the article will be submitted, gave final approval of the version to be published, and agree to be accountable for all aspects of the work.

\section{Disclosure}

The authors report no conflicts of interest in this work.

\section{References}

1. Sözen T, Özışık L, Başaran NÇ. An overview and management of osteoporosis. Eur J Rheumatol. 2017;4(1):46-56. doi:10.5152/ eurjrheum.2016.048

2. Domazetovic V, Marcucci G, Iantomasi T, Brandi ML, Vincenzini MT. Oxidative stress in bone remodeling: role of antioxidants. Clin Cases Miner Bone Metab. 2017;14(2):209-216. doi:10.11138/ccmbm/ 2017.14.1.209

3. Geng Q, Gao H, Yang R, Guo K, Miao D. Pyrroloquinoline quinone prevents estrogen deficiency-induced osteoporosis by inhibiting oxidative stress and osteocyte senescence. Int J Biol Sci. 2019;15(1):58-68. doi:10.7150/ijbs. 25783

4. Maggio D, Barabani M, Pierandrei $M$, et al. Marked decrease in plasma antioxidants in aged osteoporotic women: results of a cross-sectional study. $J$ Clin Endocrinol Metab. 2003;88 (4):1523-1527. doi:10.1210/jc.2002-021496 
5. Shao J, Zhang W, Yang T. Using mesenchymal stem cells as a therapy for bone regeneration and repairing. Biol Res. 2015;48:62. doi:10.1186/s40659-015-0053-4

6. Lin S, Lee WYW, Feng Q, et al. Synergistic effects on mesenchymal stem cell-based cartilage regeneration by chondrogenic preconditioning and mechanical stimulation. Stem Cell Res Ther. 2017;8(1):221.

7. Hass R, Kasper C, Bohm S, Jacobs R. Different populations and sources of human mesenchymal stem cells (MSC): a comparison of adult and neonatal tissue-derived MSC. Cell Commun Signal. 2011;9:12. doi:10.1186/1478-811X-9-12

8. Yoon SW, Kim DK, Kim KP, Park KS. Rad51 regulates cell cycle progression by preserving G2/M transition in mouse embryonic stem cells. Stem Cells Dev. 2014;23(22):2700-2711. doi:10.1089/scd.2014. 0129

9. Wei M, Li S, Le W. Nanomaterials modulate stem cell differentiation: biological interaction and underlying mechanisms. J Nanobiotechnol. 2017;15(1):75. doi:10.1186/s12951-017-0310-5

10. Drela K, Stanaszek L, Nowakowski A, Kuczynska Z, Lukomska B. Experimental strategies of mesenchymal stem cell propagation: adverse events and potential risk of functional changes. Stem Cells Int. 2019;2019:7012692. doi:10.1155/2019/7012692

11. Wei X, Yang X, Han ZP, Qu FF, Shao L, Shi YF. Mesenchymal stem cells: a new trend for cell therapy. Acta Pharmacol Sin. 2013;34 (6):747-754. doi:10.1038/aps.2013.50

12. Chen G, Yue A, Ruan Z, et al. Monitoring the biology stability of human umbilical cord-derived mesenchymal stem cells during long-term culture in serum-free medium. Cell Tissue Bank. 2014;15 (4):513-521. doi:10.1007/s10561-014-9420-6

13. Alves H, Mentink A, Le B, van Blitterswijk CA, de Boer J. Effect of antioxidant supplementation on the total yield, oxidative stress levels, and multipotency of bone marrow-derived human mesenchymal stromal cells. Tissue Eng Part A. 2013;19:928-937. doi:10.1089/ten. tea. 2011.0700

14. Denu RA, Hematti P. Effects of oxidative stress on mesenchymal stem cell biology. Oxid Med Cell Longev. 2016;2016:2989076. doi:10.1155/2016/2989076

15. Li Q, Gao Z, Chen Y, Guan MX. The role of mitochondria in osteogenic, adipogenic and chondrogenic differentiation of mesenchymal stem cells. Protein Cell. 2017;8(6):439-445. doi:10.1007/ s13238-017-0385-7

16. Liu SP, Ding DC, Wang HJ, et al. Nonsenescent Hsp27-upregulated MSCs implantation promotes neuroplasticity in stroke model. Cell Transplant. 2010;19(10):1261-1279. doi:10.3727/096368910X507204

17. Yang SR, Park JR, Kang KS. Reactive oxygen species in mesenchymal stem cell aging: implication to lung diseases. Oxid Med Cell Longev. 2015;2015:486263. doi:10.1155/2015/486263

18. Lee J, Cho YS, Jung H, Choi I. Pharmacological regulation of oxidative stress in stem cells. Oxid Med Cell Longev. 2018;2018:4081890. doi:10.1155/2018/4081890

19. Muthusami S, Ramachandran I, Muthusamy B, et al. Ovariectomy induces oxidative stress and impairs bone antioxidant system in adult rats. Clin Chim Acta. 2005;360(1-2):81-86. doi:10.1016/j.cccn.2005.04.014

20. Shaban S, El-Husseny MW, Abushouk AI, Salem AMA, Mamdouh M, Abdel-Daim MM. Effects of antioxidant supplements on the survival and differentiation of stem cells. Oxid Med Cell Longev. 2017;2017:5032102. doi:10.1155/2017/5032102

21. Hosnedlova B, Kepinska M, Skalickova S, et al. Nano-selenium and its nanomedicine applications: a critical review. Int J Nanomedicine. 2018;13:2107-2128. doi:10.2147/IJN.S157541

22. Khurana A, Tekula S, Saifi MA, Venkatesh P, Godugu C. Therapeutic applications of selenium nanoparticles. Biomed Pharmacother. 2019;111:802-812. doi:10.1016/j.biopha.2018.12.146

23. Alkhudhayri A, Al-Shaebi EM, Qasem MAA, et al. Antioxidant and anti-apoptotic effects of selenium nanoparticles against murine eimeriosis. An Acad Bras Cienc. 2020;92(2):e20191107. doi:10. 1590/0001-3765202020191107
24. Sart S, Song L, Li Y. Controlling redox status for stem cell survival, expansion, and differentiation. Oxid Med Cell Longev. 2015;2015:105135. doi:10.1155/2015/105135

25. Hu C, Zhao L, Peng C, Li L. Regulation of the mitochondrial reactive oxygen species: strategies to control mesenchymal stem cell fates ex vivo and in vivo. $J$ Cell Mol Med. 2018;22(11):5196-5207. doi: $10.1111 / \mathrm{jcmm} .13835$

26. Dai X, Yan X, Wintergerst KA, Cai L, Keller BB, Tan Y. Nrf2: redox and metabolic regulator of stem cell state and function. Trends Mol Med. 2020;26(2):185-200. doi:10.1016/j.molmed. 2019.09.007

27. Li J, Zuo B, Zhang L, Dai L, Zhang X. Osteoblast versus adipocyte: bone marrow microenvironment-guided epigenetic control. Case Rep Orthop Res. 2018;1(1):2-18. doi:10.1159/000489053

28. James AW. Review of signaling pathways governing MSC osteogenic and adipogenic differentiation. Scientifica. 2013;2013:684736. doi: $10.1155 / 2013 / 684736$

29. Atashi F, Modarressi A, Pepper MS. The role of reactive oxygen species in mesenchymal stem cell adipogenic and osteogenic differentiation: a review. Stem Cells Dev. 2015;24(10):1150-1163. doi:10.1089/scd.2014.0484

30. Ge C, Cawthorn WP, Li Y, Zhao G, Macdougald OA, Franceschi RT. Reciprocal control of osteogenic and adipogenic differentiation by ERK/MAP kinase phosphorylation of Runx2 and PPAR $\gamma$ Transcription Factors. J Cell Physiol. 2016;231(3):587-596. doi:10.1002/jcp. 25102

31. Kuršvietienė L, Mongirdienè A, Bernatonienè J, Šulinskienė J, Stanevičienė I. Selenium anticancer properties and impact on cellular redox status. Antioxidants. 2020;9(1):80. doi:10.3390/antiox 9010080

32. Lin CH, Li NT, Cheng HS, Yen ML. Oxidative stress induces imbalance of adipogenic/osteoblastic lineage commitment in mesenchymal stem cells through decreasing SIRT1 functions. J Cell Mol Med. 2018;22(2):786-796.

33. Liu B, Lu Y, Wang Y, Ge L, Zhai N, Han J. A protocol for isolation and identification and comparative characterization of primary osteoblasts from mouse and rat calvaria. Cell Tissue Bank. 2019;20 (2):173-182. doi:10.1007/s10561-019-09751-0

34. Yi C, Liu D, Fong CC, Zhang J, Yang M. Gold nanoparticles promote osteogenic differentiation of mesenchymal stem cells through p38 MAPK pathway. ACS Nano. 2010;4(11):6439-6448. doi:10.1021/ nn101373r

35. Behzadi S, Serpooshan V, Tao W, et al. Cellular uptake of nanoparticles: journey inside the cell. Chem Soc Rev. 2017;46 (14):4218-4244.

36. Gu H, Huang Z, Yin X, et al. Role of c-Jun N-terminal kinase in the osteogenic and adipogenic differentiation of human adipose-derived mesenchymal stem cells. Exp Cell Res. 2015;339(1):112-121. doi:10.1016/j.yexcr.2015.08.005

37. Courtial L, Picco V, Grover R, et al. The c-Jun N-terminal kinase prevents oxidative stress induced by UV and thermal stresses in corals and human cells. Sci Rep. 2017;7:45713. doi:10.1038/ srep45713

38. Almeida M. Unraveling the role of FoxOs in bone-insights from mouse models. Bone. 2011;49(3):319-327. doi:10.1016/j.bone.2011. 05.023

39. Dhoke NR, Geesala R, Das A. Low oxidative stress-mediated proliferation Via JNK-FOXO3a-Catalase signaling in transplanted adult stem cells promotes wound tissue regeneration. Antioxid Redox Signal. 2018;28(11):1047-1065. doi:10.1089/ars.2016. 6974

40. Chen D, Gong Y, Xu L, Zhou M, Li J, Song J. Bidirectional regulation of osteogenic differentiation by the FOXO subfamily of Forkhead transcription factors in mammalian MSCs. Cell Prolif. 2019;52(2):e12540. doi:10.1111/cpr. 12540 


\section{Publish your work in this journal}

The International Journal of Nanomedicine is an international, peerreviewed journal focusing on the application of nanotechnology in diagnostics, therapeutics, and drug delivery systems throughout the biomedical field. This journal is indexed on PubMed Central, MedLine, CAS, SciSearch ${ }^{\mathbb{B}}$, Current Contents ${ }^{\mathbb{B}} /$ Clinical Medicine,
Journal Citation Reports/Science Edition, EMBase, Scopus and the Elsevier Bibliographic databases. The manuscript management system is completely online and includes a very quick and fair peer-review system, which is all easy to use. Visit http://www.dovepress.com/ testimonials.php to read real quotes from published authors.

Submit your manuscript here: https://www.dovepress.com/international-journal-of-nanomedicine-journal 